\title{
A virtual scanner for teaching fundamental magnetic resonance in biomedical engineering
}

Wilhjelm, Jens E.; Duun-Henriksen, Jonas; Hanson, Lars G.

Published in:

Computer Applications in Engineering Education

Link to article, DOI:

$10.1002 /$ cae.22028

Publication date:

2018

Document Version

Peer reviewed version

Link back to DTU Orbit

Citation (APA):

Wilhjelm, J. E., Duun-Henriksen, J., \& Hanson, L. G. (2018). A virtual scanner for teaching fundamental magnetic resonance in biomedical engineering. Computer Applications in Engineering Education, 26(6), 21972209. https://doi.org/10.1002/cae.22028

\section{General rights}

Copyright and moral rights for the publications made accessible in the public portal are retained by the authors and/or other copyright owners and it is a condition of accessing publications that users recognise and abide by the legal requirements associated with these rights.

- Users may download and print one copy of any publication from the public portal for the purpose of private study or research.

- You may not further distribute the material or use it for any profit-making activity or commercial gain

- You may freely distribute the URL identifying the publication in the public portal

If you believe that this document breaches copyright please contact us providing details, and we will remove access to the work immediately and investigate your claim. 


\title{
A virtual scanner for teaching fundamental magnetic resonance in biomedical engineering
}

\author{
Jens E. Wilhjelm ${ }^{1}$ (D) | Jonas Duun-Henriksen ${ }^{1}$ | Lars G. Hanson ${ }^{1,2}$
}

${ }^{1}$ Department of Electrical Engineering, Technical University of Denmark, Kgs.

Lyngby, Denmark

${ }^{2}$ Danish Research Centre for Magnetic Resonance, Centre for Functional and Diagnostic Imaging and Research, Copenhagen University Hospital Hvidovre, Hvidovre, Denmark

\section{Correspondence}

Jens E. Wilhjelm, Department of Electrical Engineering, Technical University of Denmark, Building 349, Ørsted Plads, DK2800 Kgs. Lyngby, Denmark.

E-mail: jwil@dtu.dk

\begin{abstract}
A virtual scanner for introductory teaching in magnetic resonance imaging in biomedical engineering is presented and evaluated in a randomized trial of ultra-short and short-term learning. The results show similar performance, but indicate higher motivation, when compared with a classical approach, when class duration was identical.
\end{abstract}

\section{K E Y W O R D S}

biomedical engineering, magnetic resonance, matlab gui, signal analysis, virtual scanner

\section{1 | INTRODUCTION}

Magnetic resonance imaging (MRI) [11] is one of the four major tomographic imaging modalities applied clinically at hospitals world-wide. It is among the most important and rapidly evolving methods for diagnosing and monitoring. This is largely due to its flexibility that comes with a price of complexity. Design of measurements and quantitative analysis procedures requires a high level of technical training, and is typically conducted by biomedical engineers, physicists, or other groups with a similar level of technical insight. Extensive research in improvement of the technology is taking place at both universities, hospitals, and manufacturers of the equipment.

The physical principles and the signal processing aspects of MRI are unfortunately highly challenging for engineering students, partly because analogies to observations in everyday life are scarce compared with other imaging modalities based on ultrasound and radiation, and partly because MRI involves complex nuclear interaction, operations in Fourier space, and multi-dimensional signal processing.

In traditional class-room teaching of the physical principles of MRI, the engineering students are often introduced to the subject via the teacher's explanation with emphasis on the physics of spin dynamics followed by a description of the MRI measurement technique. In the experience of the authors, it is difficult for the students to comprehend the non-intuitive and complicated nuclear interaction and-at the same time-handle the many signal acquisition and processing aspects. Consequently, it can be unnecessarily difficult for the students to obtain an intuitive understanding of the physical aspects of MRI needed to reach deeper into the theory.

The present paper presents an efficient approach to introductory MRI education centered around a computerbased graphical virtual scanner tool designed for that purpose. The latter allows students to explore the basic principles of MRI and instrumentation relevant to MRI in a scientifically correct way. The aim is to introduce only the strictly necessary complexity for the students to understand MRI and instrumentation, initially providing a fairly complete picture, while leaving details for later. Designed to be pedagogically attractive, the presented approach can serve as an inspiring short introduction to MRI when time is scarce or as an appetizer and base for further studies of MRI.

The software was initially tailored for teaching in a specific engineering course in the physics of medical imaging [20] in which MRI is introduced along with a number of other imaging modalities-a common situation in university-level teaching. This common situation calls 
for special considerations, since the time needed for an indepth introduction to MRI is simply not available. Hence, the focus is chosen to be on a phenomenological understanding of the role of various system components rather than on core imaging physics (e.g., k-space formalism). Though the software has been designed for this particular situation, it is believed to be generally applicable for introducing MRI.

The basic idea of the software is to provide the students with a virtual MRI system, capable of simulating simple recordings but without the complexity of complete MRI systems. The system consists of magnet, gradient coils, transmitter, and receiver. Each component of the basic system is controllable, thus allowing the students to experiment with the basic principles of an MR scanner. Simplicity is favored over flexibility to limit the time needed for getting acquainted with the user interface.

The software was developed based on a thorough review of the existing literature, especially that on simulation tools: The technical, visual, and computational aspects of MRI makes it a natural candidate for computer-aided learning. Several educational MRI software packages are already available, and examples focused on MRI technology are briefly reviewed here. A number of programs [2,6,14-19] allow the user to choose sequences and imaging parameters and to generate corresponding synthetic MR images. Such programs are useful in later stages of MRI education [13], but they do not give new students insight into the basic physics, the system components, or their interconnection. A second class of educational programs including SpinPlayer [2] and the Bloch Simulator [7] illustrate the effect of time varying magnetic and radio wave fields (sequences) on spin ensembles. It is important to understand these basic methods involved in MR imaging, but they are outside the scope of the present work.

A quick overview of system components is given in the instrumentation section of the impressive e-MRI e-learning website by Hoa et al. [10]. This site supplements text-book explanations with well-chosen animations and interactive Flash-programs (Adobe Systems, San Jose, CA) to illustrate key concepts. The section on instrumentation is promoted in the basic physics part of the course. The focus is similar to that of the present work, but the approach is quite different. A traditional bottom-up approach is taken on the e-MRI site in the sense that the learning path starts at basic principles, passes over system components, signal formation, and imaging methodology to images. Similar paths are followed with varying degrees of graphics, interactivity, and detail in other web-based presentations, for example [11]. Such approaches are particularly good if there is sufficient time available, but it is useful with an alternative or supplemental top-down, interactive approach that gives the students a quick overview of the key components of MRI and their immediate role in imaging, while details are postponed for later. The software presented here thus fills a gap in the educational software portfolio.

\section{2 | LEARNING OBJECTIVES}

The objectives of the teaching process described in the present paper are that the students shall learn the basic principles and how they are brought to use-from a phenomenological point of view. Specifically, a student who has successfully met the objectives will be able to:

- Explain the interaction between the radio waves and polarized sample, and the resulting magnetic resonance behavior of the nuclei under investigation.

- Describe the major components of an MR scanner and their roles.

- Identify what is needed to obtain a signal (observe a magnetic resonance response) from an object.

- Identify which parameters are relevant for obtaining a response as well as what the parameters represent.

- Identify the ranges of typical values of these parameters.

- Explain how these parameters are related (equations, dependence).

- Sketch the transmitted and received signals in an MR scanner.

\section{3 | DESCRIPTION OF VIRTUAL SCANNER}

The virtual scanner [4] was designed in order to fulfill the above listed learning objectives. It was developed in MATLAB (version R2017b, The MathWorks ${ }^{\mathrm{TM}}$, Natick, MA) running under most PC operation systems, including Linux, macOS ${ }^{\mathrm{TM}}$, and Microsoft Windows ${ }^{\mathrm{TM}}$. MATLAB is used by the primary target students throughout their education. It offers relatively simple design of graphical user interfaces, and its use may inspire students (they have access to the source code). This section describes the design goals followed by an explanation of how-and with which motive - the different parts of the virtual scanner were made.

\section{1 | Design goal}

Since there is limited time in the course for MRI, it is not possible for the students to learn the mathematical description of the resonance phenomenon in detail. Thus, it is deemed better to make the students understand it from a phenomenological, classical view [8].

In making the software, the design goals were primarily to produce a virtual scanner that could give an intuitive understanding of the use of the main components involved 
in MRI and how they operate together. The virtual scanner had to be graphical to facilitate recognition and visual learning while important formulas should be represented as well. Furthermore, it was important that the program responded quickly so that the students do not get impatient and divert attention from the learning objectives.

\subsection{Elements of the virtual scanner}

Based on the learning objectives in section 2, the main components of the virtual scanner were designed as shown in Figure 1. As illustrated, the graphical user interface is divided into five regions: Upper-left: Visualization of physical object space. Lower-left: Control panel to adjust settings and parameters for the scanner. Upper-right: Transmitted and received signal, respectively. Lower-right: The reconstructed image. Lowest right: This panel features Reset and Help buttons as well as About buttons for instructions to the program.

\subsection{1 | Control panel}

The main interaction with the program takes place through the control panel at the lower left corner of Figure 1. The different components, Magnet, Gradient, RF pulse transmitter, and Object, can be switched on and off. By doing that, the component or its effect(s) will be visualized in the physical object space in the upper left corner. At the same time, the program will check if there will be a measurable signal from the object. If so, signals are shown in the upper right corner. If all conditions are met, and an image can be reconstructed, this will be shown in the lower right corner.

In the control panel, different settings for the components can be applied. As an example, consider changing the main magnetic field, $B_{0}$, from 0 to $3 \mathrm{~T}$. This will in turn change the Larmor frequency, given by

$$
f_{0}=\gamma B_{0}
$$

where $\gamma$ is the gyromagnetic ratio, which is approximately $42 \mathrm{MHz} / \mathrm{T}$ for protons. The result of this equation is evaluated and shown in the control panel, so that the frequency of the RF pulse transmitter, $f_{\mathrm{p}}$, can be adjusted and related to the Larmor frequency. For the RF pulse transmitter, it is also possible to adjust the amplitude, the pulse duration, $t_{\mathrm{p}}$, the repetition time, $T_{\mathrm{R}}$, and the echo time,

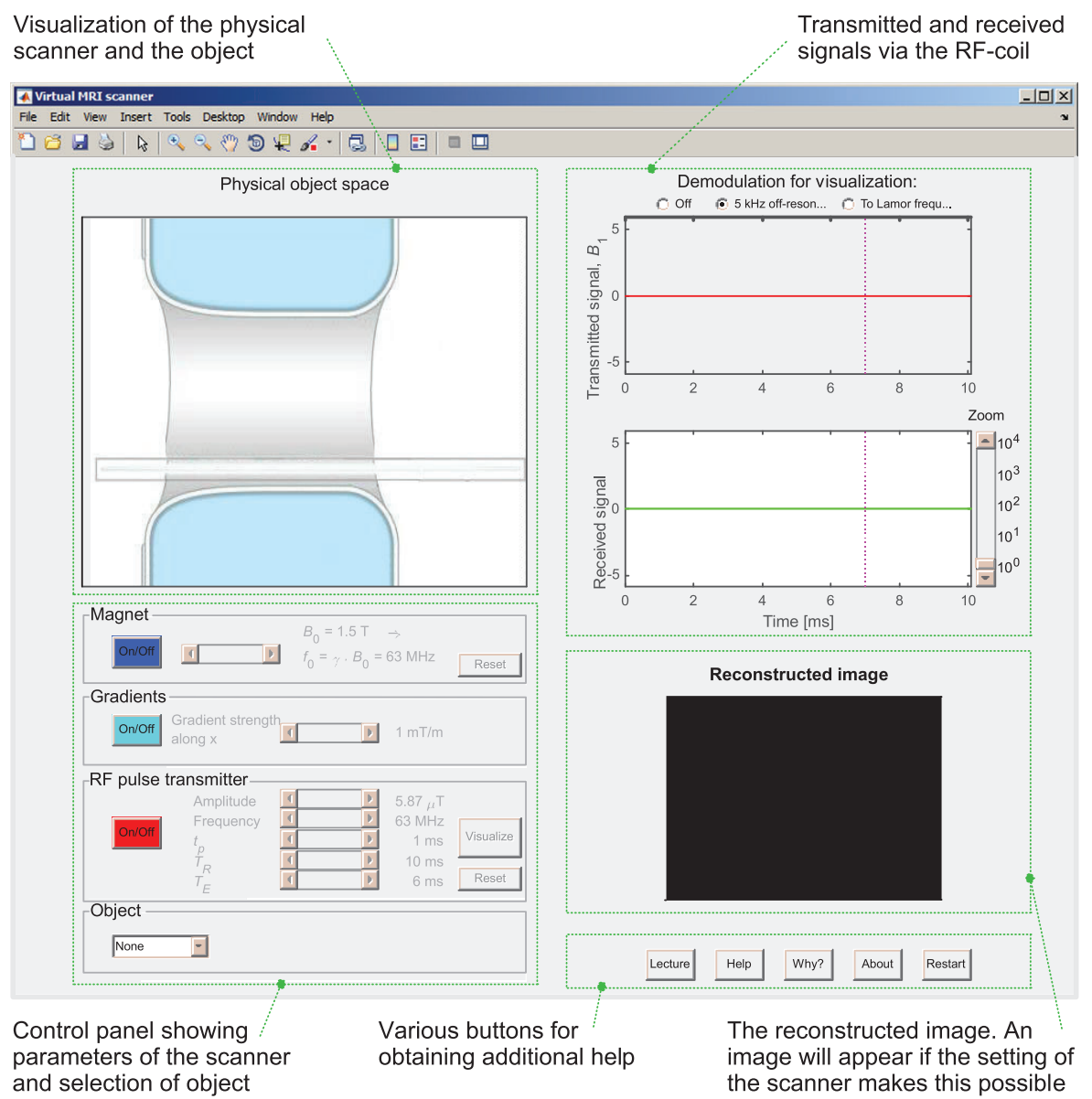

FIGURE 1 The virtual scanner as it appears when first started. The window is divided into five regions as indicated with green boxes 
$T_{\mathrm{E}}$. The latter is defined as the duration between excitation and effective signal read-out time, for example, the time before origo in k-space is reached. By hitting the Visualize button, the meaning of these parameters will be shown on the transmitted signal in order to give a graphical understanding, as illustrated in Figure 2. Alternatively, information can be obtained by holding the mouse cursor over one of the buttons or sliders: A yellow tool tip box will appear, explaining the effects of the control.

Another thing the students can alter is the object within the scanner. This object has the shape of a mannequin. It is assumed that this mannequin is either a homogeneous phantom or an actual person. The homogeneous substances can be water, ice, oil, fat, bone, muscle, cartilage, or gold. These are chosen to allow seeing the influence of material properties on the MR signal. An approximate $T_{2}$ relaxation time is given for each of the substances, except for the heterogeneous body of a person, since no single $T_{2}$ relaxation time can be specified. For the non-magnetic substance gold, $T_{2}$ has been set to zero.

\subsubsection{Visualization of scanner components}

When the different scanner components are switched on at the control panel, they will appear in the physical object space. The magnet and gradient coil systems are merely visualized as blocks, while the RF pulse transmitter is shown as a coil surrounding the object, as illustrated in Figure 3. The main magnetic field is chosen vertical, which is normal for open scanners. The chosen coil design is not conventional for a whole body scanner but it is well-known for engineering students, and is useful for illustration of the sensitivity to flux variations orthogonal to the main magnetic field. When the magnet is on and $B_{0}$ is different from zero, arrows showing the direction of the magnetic field will appear between the two magnets. If the gradient is also on, the arrows will vary in length depending on their position in the gradient field (the effect is strongly exaggerated for visualization purposes). Obviously, a gradient along one direction is not enough for imaging. Nevertheless, the program provides images even though only slice selection is simulated so that the students can gain an understanding of the gradient-concept, without

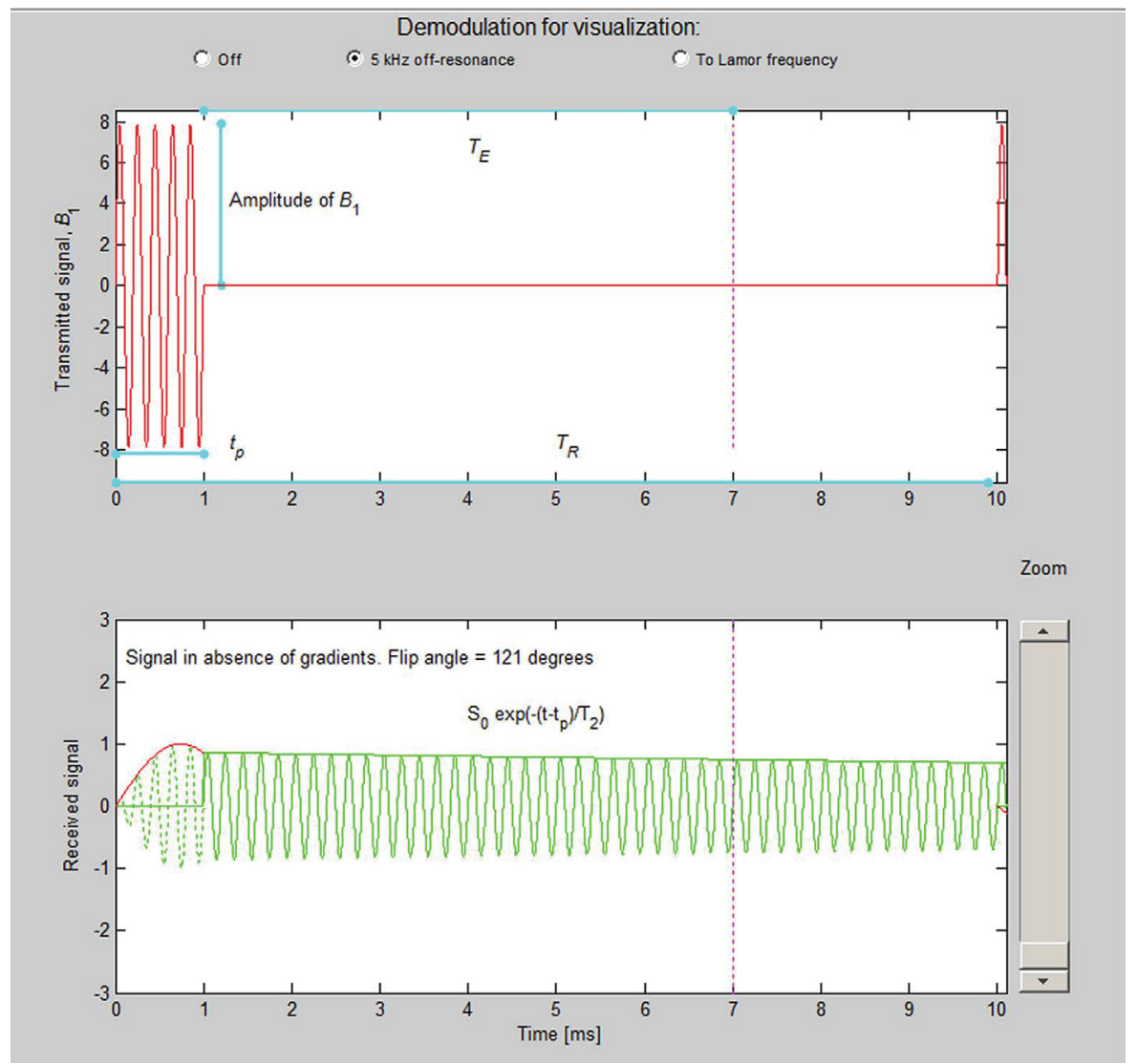

FIGURE 2 Top panel: Transmitted signal (square pulse) with graphical illustration of different pulse parameters. Bottom panel: Illustration of the received signal. The red curve shows the undetected signal during excitation. The signal amplitude is proportional to the sine of the flip angle that increases linearly during excitation. A quarter of a full sine wave corresponds to $90^{\circ}$. In this figure, the amplitude, $B_{1}$, is $7.87 \mu \mathrm{T}$, and the excitation duration, $t_{\mathrm{p}}$, is $1 \mathrm{~ms}$ 


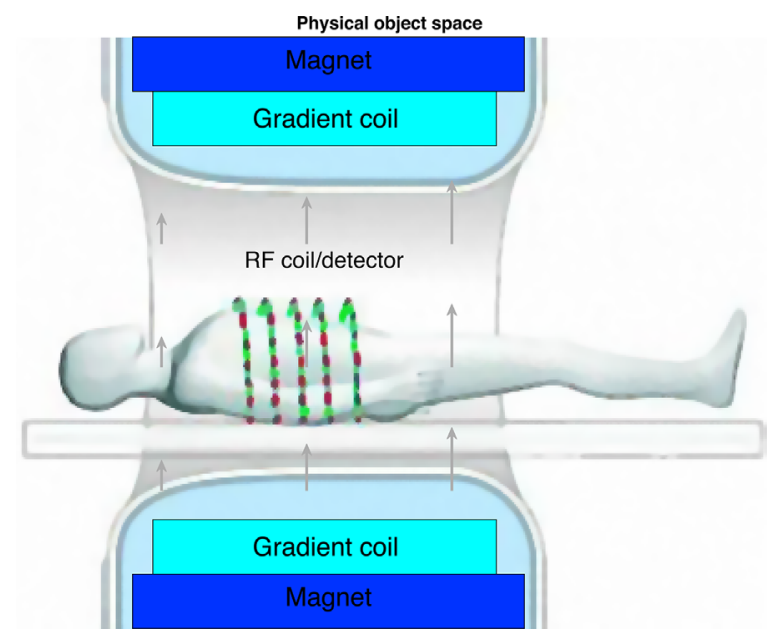

FIGURE 3 The visualization of the scanner and the object when all scanner components are active

having to understand how gradients are used to get in-plane resolution, which is outside the scope of the program.

\subsection{3 $\mid$ Transmitted and received signals}

The upper right corner of Figure 1 shows the transmitted RF pulse (or a demodulated version of this). It only takes an RFcoil with an amplitude and frequency different from zero, before a transmitted signal will be seen, regardless of the presence of a magnetic field, a gradient, or an object in the scanner.

Just below the transmitted signal, the received signal is shown, also called the FID signal (free induction decay). This is somewhat more complex. It is an aim of the virtual scanner to make the students understand when the signal will be different from zero, and how the different parameter settings affect it. To measure a signal, it is necessary to excite the protons in the object first. This is done by positioning the body in a magnetic field, calculate the Larmor frequency, and then excite the protons with radio waves of that frequency. The RF field rotate the nuclei away from equilibrium. After application of an RF pulse of duration $t_{\mathrm{p}}$, the net-magnetization will be rotated by a flip angle given by the following formula:

$$
\alpha=\gamma B_{1} t_{\mathrm{p}}
$$

where $B_{1}$ is the amplitude of the transmitted RF-signal. This is illustrated in the graph that also shows the calculated flip angle, as seen in Figure 2.

The amplitude of the received signal, $V$, is normalized to the maximum obtainable signal at a flip angle of $90^{\circ}$ following full relaxation. It is $T_{1}$-weighted corresponding to the repetition time $\left(T_{\mathrm{R}}\right)$ employed and it decreases exponentially according to:

$$
V \propto \exp \left(-T_{\mathrm{E}} / T_{2}\right)
$$

where $T_{\mathrm{E}}$ is the echo time (field inhomogeneity is ignored). The transmitted and received signal can be shown in three different demodulation modes, as illustrated in Figure 4. If no demodulation is done, the oscillations of the RF-signals are shown with the "true" frequencies. If the RF-frequency is at $63 \mathrm{MHz}$ corresponding to the Larmor frequency for a $1.5 \mathrm{~T}$ scanner, the oscillations are so quick that they will not be separable on the time scale used. Instead, a demodulation $5 \mathrm{kHz}$ off-resonance can render the nature of the signal more comprehensible. Finally, it is also possible to demodulate the signals at the Larmor frequency.

In summary, a received signal is shown when the $B_{0}$ field is turned on, there is a magnetic object present and the excitation field is present and tuned to the Larmor frequency.

\subsection{4 $\mid$ The reconstructed image}

The reconstructed image is shown in the lower right hand corner, if the settings of the scanner permit this. Noise is added, and the edges are blurred to make the image more realistic as seen in Figure 5. When the parameters are set correctly for human imaging and a human thorax consequently appears as illustrated in Figure 5, the image changes with the settings of pulse width, RF frequency and gradient strength to reflect which cross-section of the object is being imaged.

In summary, a reconstructed image is shown, if the condition for showing a received signal is present and the transmitted signal $B_{1}$ is giving a flip angle different from zero and the gradient is turned on.

\subsection{5 | Help}

Pressing this button will explain the program and also provides a hint, when relevant, as to why an MR image is not displayed.

\section{4 | ASSESSMENT OF LEARNING POTENTIAL}

An attempt to evaluate the virtual scanner was conducted as a randomized trial by exposing half of the class to a classical lecture approach while-simultaneously - the other half was exposed to an approach involving the virtual scanner.

\section{1 | Materials and methods}

\subsection{1 $\mid$ Course settings and environment}

The evaluation was performed in the first of three classes on MRI in the course 31540 Introduction to medical imaging [20] 


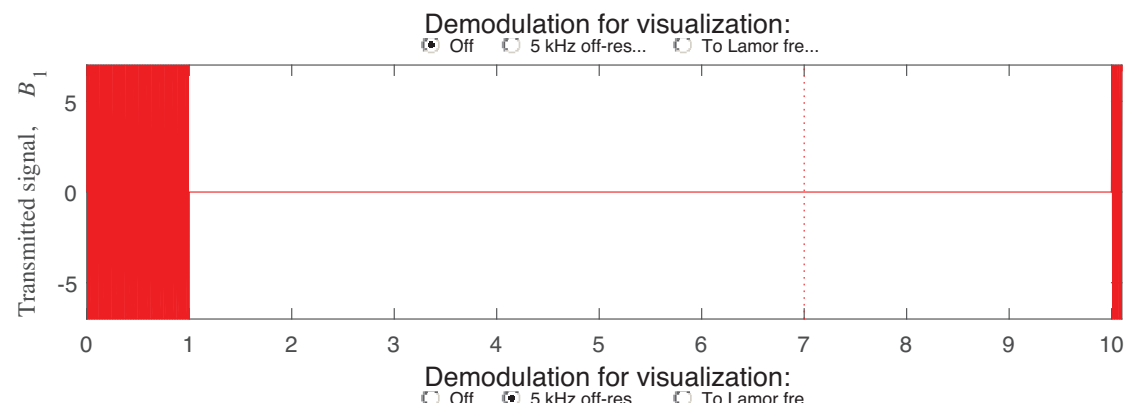

(a)

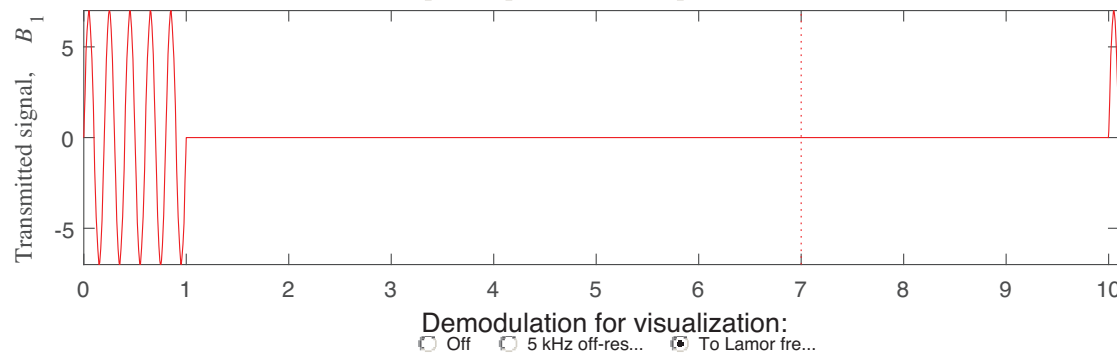

(b)

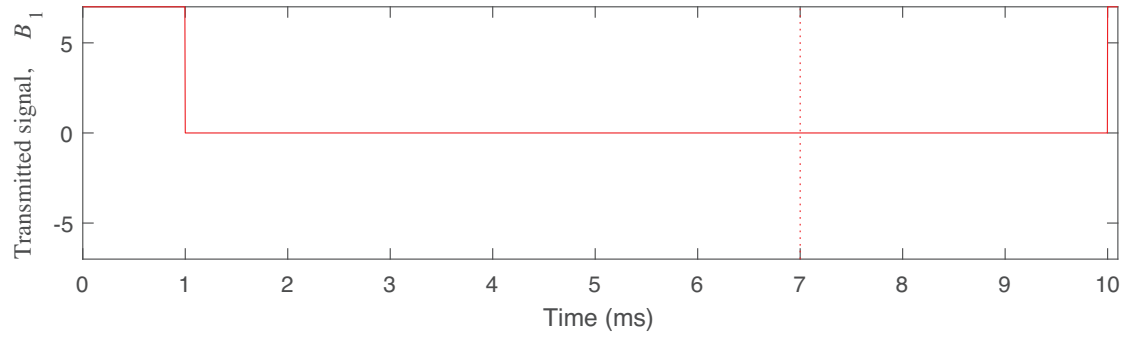

(c)

FIGURE 4 The transmitted signal for $T_{\mathrm{R}}=10 \mathrm{~ms}$ and three different modes of modulation. (a) The transmitted signal without demodulation. Since the frequency of the RF-waves is $63 \mathrm{MHz}$ in this example, it is not possible to see the oscillations. (b) The transmitted signal is demodulated to $5 \mathrm{kHz}$. (c) The demodulation is set to zero hertz. All vertical axes are relative

taught at the 5th semester of the Bachelor's program in Biomedical Engineering at the Technical University of Denmark and Faculty of Health and Medical Sciences, University of Copenhagen. The course is also offered to students in electrical engineering and similar programs. Besides MRI, the course also teaches ultrasound, planar Xray, computed tomography, and positron emission tomography. The course runs once a week in an afternoon module from 13:00 to 17:00 hours over a period of 13 weeks.

The course was attended by 40 students from this bachelor program and seven other students mainly from abroad. The comparison of the two approaches was performed in the auditorium (classical) or in the data bar (virtual scanner), both of which the students were highly accustomed to.
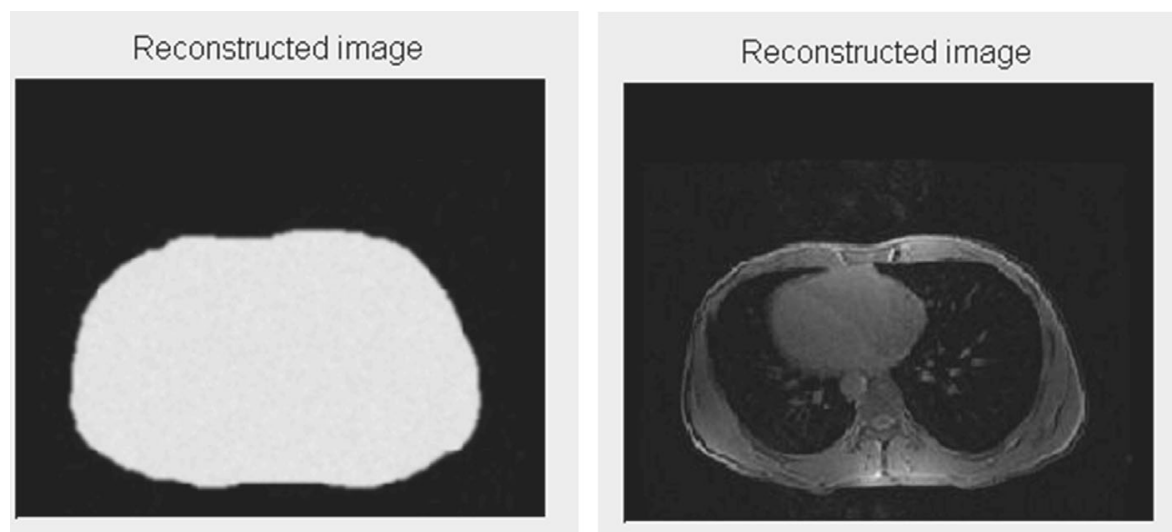

FIGURE 5 Reconstructed images for a body-shaped, water-filled mannequin (left panel) and a human thorax (right panel) 


\subsection{2 | Quiz taken prior to and after the class}

A multiple-choice quiz of 16 questions with one correct answer, three distractors and one "Do not know" answer was taken by the students both before and after the class. Most of the students have encountered this type of quiz before.

The quiz was made to test the learning objectives in subsection 2 on a level that corresponded to the first lecture; consequently, the complexity of the questions corresponded to the first levels on Bloom's taxonomy [1]. Thus, the quizzes primarily measured improvement in knowledge and to a lesser degree understanding due to the two learning approaches. Specifically, the central equation and the associated physical aspects listed in the learning outcomes that the students were supposed to learn during either of the classes-and therefore attempted tested by the quiz-are illustrated in Figure 6 .

The students were asked to write their name on the answer sheet, but were also informed that the purpose of the quiz was to perform a comparative evaluation of the two teaching approaches and that the results of the quizzes did not count toward the final grade.

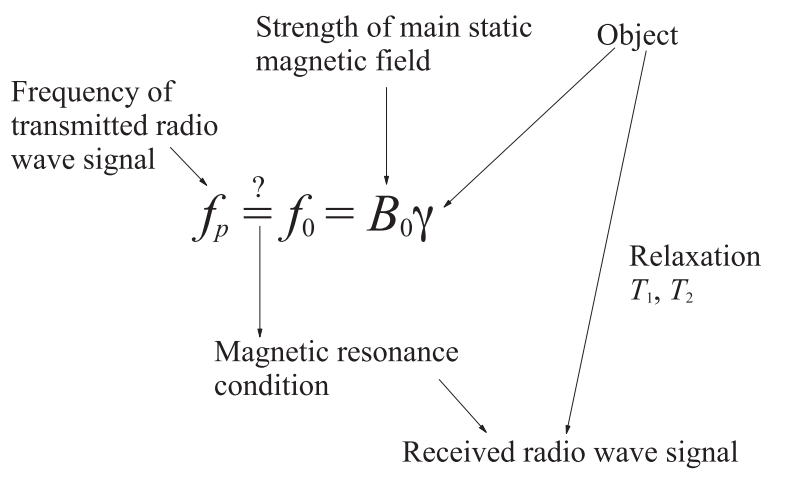

FIGURE 6 Example of parameter and component relations that the program and the accompanying guide are intended to convey to the students
We hypothesized that the students scored quite low in the quiz taken before class and high when taking the same quiz after class. For the quiz taken after class, we also hypothesized that the students attending the virtual scanner class scored relatively better than the students attending the classical lecture class.

\subsection{3 $\mid$ Comparison procedure}

The week prior to this evaluation, students were informed in plenum about the possibility of participating and they were asked if they wanted to (all agreed to participate). This gave some sort of co-ownership of the trial and therefore probably a more positive attitude toward following the rules set up.

One day before the evaluation, the students of the course were randomly divided into two equally large groups of 24 students (10 male and 14 female) in the classical group and 23 students (11 male and 12 female) in the virtual scanner group. The students worked in teams in the course, but the selection was blind to the constitution of these teams. There were approximately an equal amount of international students in both groups.

The flow of the trial is shown in Table 1. Details on the different parts of the classes can be found in the Appendices revealed by superscript in the table.

In both groups, the teaching language was English. The lecturer and instructor were different, since the teaching took place simultaneously.

\section{5 | RESULTS}

Of the 47 students, 22 students participated in the classical approach while 18 students participated in the virtual scanner approach. The remaining seven students either did not show up, showed up too late to participate or erroneously selected

TABLE 1 Overview of the teaching where half of the students followed a traditional lecture simultaneously with the other half using the MRI virtual scanner

\begin{tabular}{|c|c|c|}
\hline Flow & Classical approach & Virtual scanner approach \\
\hline Pre quiz (10 min) & \multicolumn{2}{|c|}{ MC quiz } \\
\hline Main learning activity (40 min) & $\begin{array}{l}\text { Classical lecture in lecture room: } \\
\text { - Compass magnetic resonance } \\
\text { - Comparing vibration with precession }{ }^{\text {I.ii }} \\
\text { - Larmor frequency } \\
\text { - Transmission \& reception } \\
\text { - Spatial information (1D) })^{\text {I.ii }} \\
\text { - Example of system components }\end{array}$ & $\begin{array}{l}\text { Individual work in databar: } \\
\text { - A short lecture }(<10 \text { min) on compass } \\
\text { magnetic resonance }{ }^{\mathrm{I} . \mathrm{i}} \text { and introduction } \\
\text { to the GUI of the MRI virtual scanner } \\
\text { - Use of the MRI virtual scanner in } \\
\text { order to try to answer the questions in } \\
\text { the guide } \\
\text { - Teacher present to answer possible } \\
\text { questions }\end{array}$ \\
\hline Post quiz (10 min) & \multicolumn{2}{|c|}{ Same MC quiz } \\
\hline
\end{tabular}


the wrong group, despite the clear instruction of where they were supposed to show up. These seven students were removed from the analysis.

Despite extensive testing and planning prior to the trial, some students reported that MATLAB stopped responding, requiring reboot of the computer that they were working with. A subsequent analysis of the login data for the entire data-bar showed that at least five students had to reboot their machine during the experiment. Consequently, those students did not have as much time with the virtual scanner as planned.

\section{1 | Ultra-short term learning effect}

The answers to all four quizzes are given in Figure 7. The correct answer is indicated above the column. Blank answers were counted as "Do not know," denoted by letter E. It is observed for both approaches, that the number of "Do not
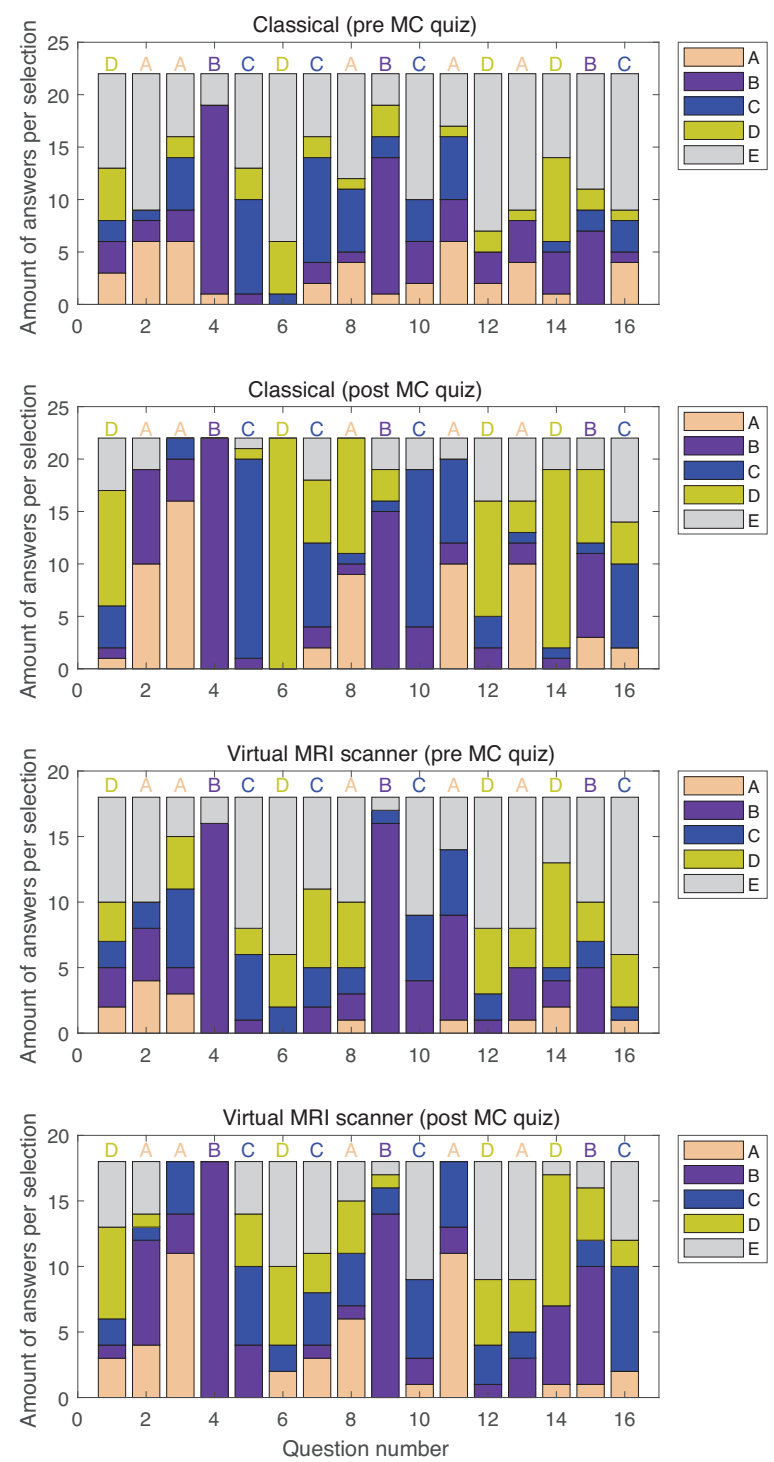

FIGURE 7 Overview of all test answers. Blank is depicted as "E" know" answers decreased from the pre quiz to the post quiz. The answers, however, did not always become correct: In the classical class, there were 37 cases of the "Do not know" answer changing into a wrong answer. In 13 cases, a correct answer was changed into a wrong. The corresponding figures for the virtual scanner class were 26 and 18, respectively.

Comparing the two pre-quizzes, there is some amount of differences in the answer pattern for some problems (e.g., 7, 11, and 12). For the post quizzes, there are remarkable differences in the students' answers to problems 5, 6, 13 . These problems all relate to the Larmor equation, which was one of the most central parts of the classical lecture.

Figure 8 summarizes all the data from Figure 7. When comparing the results of the two pre quizzes, it is observed that the students of the virtual scanner approach perform slightly worse compared with those that followed the classical approach; however, the difference is not statistically significant $(p=0.24)$. The improvement from pre to post quiz was statistically significant for the classical class $(p=0.003)$, but not for the virtual scanner class $(p=0.07)$.

The above can be observed in more detail from Figure 9 which shows the relative amount of all possible combinations of answers in the pre and post quizzes. Specifically, the main differences between the two groups are the following:

- The virtual scanner group has a relatively higher number of wrong and "Do not know" answers that remain unchanged when going from the pre quiz to the post quiz. And, likewise, the number of correct answers that stayed correct is lower for the virtual scanner class compared with the classical class.

- The relative number of "Do not know" answers that became correct is nearly twice as large in the classical approach compared with the virtual scanner approach.

- The relative number of correct answers that became wrong is more than $50 \%$ larger in the virtual scanner approach.

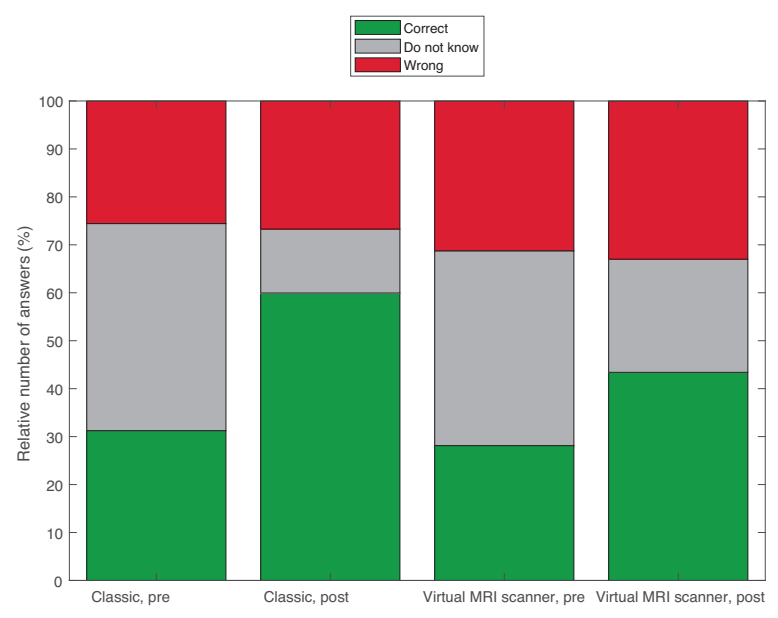

FIGURE 8 The combined results of Figure 7 

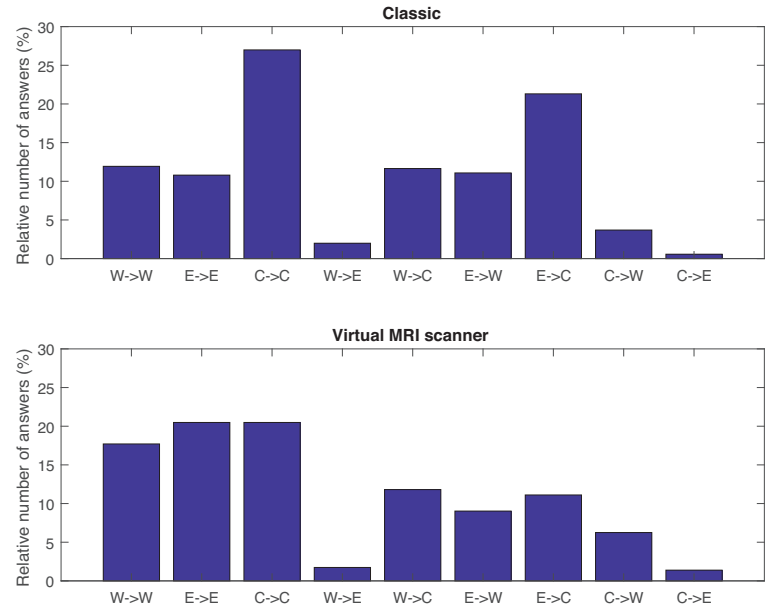

FIGURE 9 Relative number of different combination of answers from pre quiz to post quiz for both learning approaches. $\mathrm{W}=\mathrm{Wrong}$, $\mathrm{E}=$ "Do not know", $\mathrm{C}=$ Correct

The different transition pairs likely have causes beyond randomness from guessing. Such causes are outlined in Table 2.

\subsection{Short-term learning effects}

The results showed until now have been concerned with the ultra short-term learning effects. The short-term learning effect was also explored for the two groups of students. Specifically, the results of the final multiple-choice exam, placed months later, were investigated for both groups of students (both groups missed one student that for unknown reasons did not participate in the exam). All 25 problems in this exam were categorized into three groups:

- Problems that contained only the MRI modality (three problems)

- Problems that contained MRI in combination with other modalities (three problems)

- The entire exam of 25 problems.

Figure 10 show the relative numbers of correct, "Do not know" and wrong answers in these three categories. From the bar plot, and a statistical analysis performed on the raw data, it

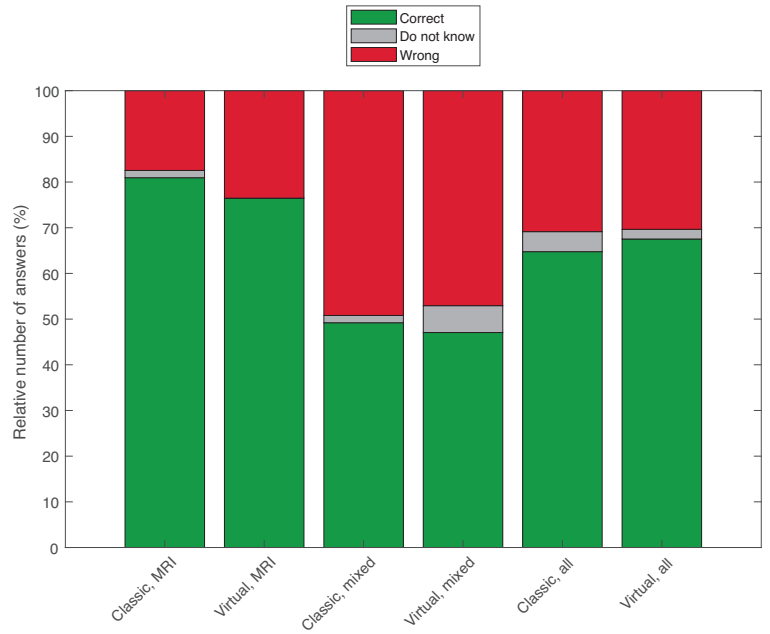

FIGURE 10 The students' answers to the three categories of problems in the final multiple-choice exam for those students that participated in the classical class and in the virtual scanner class. The problems were organized in three groups: "MRI"= problems only considering MRI. "mixed" = problems containing MRI and at least one additional modality. "all" = The entire exam

was found that none of the small differences observed were statistically significant.

\section{3 | Short-term motivation effects}

Finally, on a time scale similar to the one for the exam of the course, it was investigated to which degree there were possible differences in the way the students were motivated for further work with MRI. This was based on the final report, that each of the 11 teams had to hand-in at the end of the course, 1 week prior to the exam (the report counted toward the final grade with $40 \%$ ). Specifically, the students of the teams typically distributed the chapters of the report between them including the theoretical chapters on the five imaging modalities covered by the course. Therefore, in the report, the students were required to self-declare the contributions of each of the approximately four team members. From the 11 reports, it was found that three students from the classical class wrote the MRI chapter while six students who had attended the virtual scanner class wrote the MRI chapter. Two reports did not provide any self-declarations.

TABLE 2 Stipulated learning effect derived from their answers to the pre and post quizzes

\begin{tabular}{lllll} 
& & \multicolumn{2}{c}{ Post quiz } \\
\cline { 3 - 5 } \multicolumn{1}{c}{ Answer } & Correct & Do not know & Wrong \\
\cline { 3 - 5 } Pre quiz & Correct & Knows in advance & Learning has made the student uncertain & No learning (e.g., misunderstanding) \\
& Do not know & Learning & Serious about own limitations & No learning (e.g., misunderstanding) \\
& Wrong & Learning & Class did lead to awareness of limitations & No learning. Neither did class lead to higher \\
& & & & self-awareness of own limitations
\end{tabular}




\section{6 | DISCUSSION}

\subsection{Initial observations}

Initially, a few aspects of the randomized trial turned out to be in disfavor to the virtual scanner approach: Some students were delayed by technical problems with MATLAB requiring reboot of the computer they worked with. This biased the investigation since such problems did not occur in the classical approach. Some students tried to understand the virtual scanner by actually considering the source code (the m-files). This is indeed possible, but due to the complexity of the software, this is quite time consuming and probably not a favorable way to obtain insight in a 40 min class. This unintended behavior has also been observed by others [3]. Finally, the students need time to familiarize themselves with the interface adding an extra obstacle to the learning process compared with the classical approach.

Another aspect of measuring performance with tests just prior to and after a relatively short activity is that it might give bias in favor of the classical approach. Specifically, the students of the classical approach should provide answers to questions where they have just heard or seen information that directly lead to the answer compared with the situation for the students of the virtual scanner approach, who should provide answers to aspects that they might have learned via the virtual scanner (since, during the available $40 \mathrm{~min}$, it cannot be guaranteed that all aspects are considered by the students). This might be a pronounced effect for students with a good short-term memory.

Finally, while a multiple-choice quiz is very appealing since it is fast, convenient and quantitative, it is very difficult to produce a balanced set of problems that is fair to both teaching approaches.

\section{$6.2 \mid$ Analysis of results of trial}

The answer pattern in Figure 8 to the two pre quizzes appear quite similar, however, the number of correct answers is slightly lower and the number incorrect answers slightly higher for the virtual scanner class (not statistically significant). The number of "Do not know" answers accounts for more than $33 \%$ of the answers for both classes, suggesting that the students are aware of own limitations.

When comparing the pre quiz with the post quiz for both classes, the following can be observed: Both the number of correct answers as well as the number of incorrect answers increase at the expense of the number of "Do not know" answers. This is surprising and suggests that the students believe they know more than they do (overly confident) or have misunderstood certain concepts.

Comparing the post quizzes it is observed that for the classical class, the relative number of correct answers is higher and the relative number of wrong answers is lower, suggesting that the students of the classical class gained a somewhat higher ultra-short term learning.

When comparing Figure 9, it is seen that four columns exhibit a noticeable difference in performance between the two approaches: "Wrong to Wrong," "Do not know" to "Do not know," "Correct" to "Correct," and "Do not know" to "Correct" are all in favor of the classical approach. If the stipulated effect in Table 2 applies, the results suggest that ultra-short term learning is somewhat less in the virtual scanner approach.

This is aligned with the observation that the improvement of the classical class was statistically significant while this was not the case for the virtual scanner approach. However, taking into account the unintended handicap present with the virtual scanner approach, a conservative estimate would be, that the two methods roughly work equally well.

Despite the fact that the learning appears to be lower for the virtual scanner class-when measured as ultra-short time learning - this effect is not present at the final exam (i.e., the results of Figure 10 which were not statistically significant). This may be attributed to a higher gain in motivation by the students that undertook the virtual scanner class, further supported by the observation, that twice as many MRI chapters in the final reports were made by students who undertook the virtual scanner class. This is noteworthy, especially because this chapter is considered the most difficult of the entire report.

The statistical analysis was performed with a two-sample $t$-test; even though relatively robust to the distribution of the data, the assumption of a normal distribution of the data tested was not met. Since two educational methods that are both believed to be good, were compared, and since the sample is relatively small and inhomogeneous, the lack of statistical difference is not surprising and expected independent of the chosen statistical method, but importantly, the results indicate that neither method is much worse than the other.

\section{3 | Conclusions regarding the trial}

The randomized trial was designed to ensure that both approaches had the same amount of confrontation time, since (teacher) time was the limited resource. A class duration of $40 \mathrm{~min}$, however, might not be the optimal setup for the virtual scanner since one of the advantages of the virtual scanner is that the students are likely motivated to explore and work independently and longer compared with the classical approach. Specifically, the classical approach consisted of self-study (reading) and a lecture. Here after, the students might leave the learning path. In the virtual scanner, the students might be involved with learning for a longer time, since the scanner interface together with the provided questions (subsection I.iii) clearly expose to the students 
what they have not yet explored, which is assumed to tempt their curiosity. Thus, students engaged with the virtual scanner might be more eager to work longer with the subject, compared with students following the classical approach.

The above combined with the observations of aspects in disfavor of the virtual scanner approach (see section 6.1) renders it plausible that the two learning approaches roughly work equally well, when the same amount of time is available. However, understanding the topics by the virtual scanner class means that they have worked more actively with the material than in the classical approach rendering it more probable that they obtain a higher increase in lasting gain of competences compared with the classical approach.

\section{7 | IMPLEMENTATION DETAILS SPECIFIC TO THE VIRTUAL SCANNER}

In order to ensure that most students use the program intensively, it is important that it functions well, is easy to understand and is clearly and logically organized. If not, there is a risk that the student gets lost, wastes precious time and loses motivation. Simulation flexibility was therefore traded off for a simple and intuitive user interface.

In order to appeal to as many students as possible, three levels of demodulation of the transmitted and received signals can be visualized. This can improve the understanding for students that have not met these concepts in signal analysis.

There are some technical limitations of the virtual scanner: Whereas students can learn the basics of slice selection and gain experience with parameters influencing this, the mechanisms used to create an MR image is not covered by the virtual scanner, since this would introduce complexity beyond the scope of the course. Finally, relaxation during excitation as well as field inhomogeneities are ignored.

Since the randomized trial was conducted, the virtual scanner has been used routinely in the course and with more time allowed for the students with the presence of instructors. Unfortunately, this has not been cost-free: Today, it is not used on a single type of computer (data-bar) but rather on a larger number of laptop computers with different operating systems and different versions of MATLAB, which sometimes affect the functionality. Finally, the screen size on some of the lap-top computers is occasionally too small to allow optimal visualization. These factors have the potential of limiting engagement for some students.

MATLAB was chosen since it is already used by the students, and is well suited for implementation including GUI design. Licensing is costly, however, and access somewhat limited. A choice for future reimplementation may be one of the widely used alternatives to MATLAB (e.g., Python), or HTML5 [12]/JavaScript [5], which can make the simulator work within any modern browser.

\section{8 | CONCLUSIONS AND PERSPECTIVES}

This work presented a virtual MRI scanner for use in biomedical engineering education. It is implemented in MATLAB as a graphical user interface showing both the physical object space as well as the operator control panel, settings, signals and images. It was tested in a non-blinded randomized trial against a classical approach and the results show-when taking into account the obstacles present for the virtual scanner approach - that the two methods performed roughly equal. An attempt to assess how motivated the students were for further work with MRI showed that in the contribution to the final report of the course, twice as many students following the virtual scanner approach wrote the challenging MRI chapter compared with students that undertook the classical approach.

The main pedagogical advantage of the virtual scanner is that it likely stimulates more activity and engagement and thereby eventually provides more learning than the classical approach. In addition, the virtual scanner also added to the palette of learning tools available for the student to engage in his/her own personalized learning style.

\section{ACKNOWLEDGMENTS}

The contributions from Elna Sørensen, Allan Jørgensen, and Nina Kjærgaard, DTU Elektro, Markus Nowak Lonsdale, Dept. of Clin. Physiol. \& Nucl. Medicine, Copenhagen University Hospital, Bispebjerg Hospital and Michael May and Pernille Rattleff, LearningLab·DTU are greatly appreciated.

\section{ORCID}

Jens E. Wilhjelm (D) http://orcid.org/0000-0002-9367-8421

\section{REFERENCES}

1. L. Anderson et al., A taxonomy for learning, teaching, and assessing: A revision of bloom's taxonomy of educational objectives, Addison Wesley Longman, Inc., USA, 2001.

2. H. Benoit-Cattin et al., The SIMRI project: A versatile and interactive MRI simulator, J. Magn. Reson. 173 (2005), 97-115.

3. R. D. Burke et al., A virtual engine laboratory for teaching powertrain engineering, Comput. Appl. Eng. Educ. 25 (2017), 948-960.

4. J. Duun-Henriksen, L. G. Hanson, and J. E. Wilhjelm, A graphical simulator for MRI. Available online at: http://bme.elektro.dtu.dk/ jw/webbook/?MRI/demos/main.html. Accessed 5 January 2018.

5. ECMAscript Language Specification, ECMA International, 19972017. Available online at: https://www.ecma-international.org/ publications/standards/Ecma-262.htm. Assessed 6 April 2018. 
6. T. Hackländer and H. Mertens, Virtual MRI: A PC-based simulation of a clinical MR scanner, Acad. Radiol. 12 (2005), 85-96.

7. L. G. Hanson, A graphical simulator for teaching basic and advanced MR imaging techniques, Radiographics 27 (2007), e27.

8. L. G. Hanson, Is quantum mechanics necessary for understanding magnetic resonance? Concepts Magn. Reson. 32A (2008), 329-340.

9. L. G. Hanson, A classical explanation of magnetic resonanceSoftware and animations illustrating basic MR concepts. Available online at: http://www.drcmr.dk/MR

10. D. Hoa, A. Micheau, and G. Gahide, Creating an interactive webbased e-learning course: A practical introduction for radiologists, Radiographics 26 (2006), e25.

11. J. P. Hornak, The Basics of MRI. Available online at: http://www. cis.rit.edu/htbooks/mri/. 2010. Accessed January 2018.

12. HTML5, W3C Recommendation, 2014-2017. Available online at: https://www.w3.org/TR/html52/. Assessed 6 April 2018

13. E. Kanal and M. W. Perlin, Computer-based tutorial in $M R$ imaging, AJNR Am. J. Neuroradiol. 13 (1992), 1527-1524.

14. F. Liu et al., Fast realistic MRI simulations based on generalized multi-pool exchange tissue model, IEEE Trans. Med. Imaging 36 (2016), 527-537. https://doi.org/10.1109/TMI.2016.2620961

15. R. B. Lufkin et al., MRI simulator for instruction in pulse-sequence selection, AJR Am. J. Roentgenol. 147 (1986), 199-202.

16. M. B. E. Olsson, R. Wirestam, and B. R. R. Persson, A computer simulation program for MR imaging: Application to RF and static magnetic field imperfections, Magn. Reson. Med. 4 (1995), 612-617.

17. D. Rundle et al., Magnetic resonance imaging simulator: A teaching tool for radiology, J. Digit Imaging 3 (1990), 226-229.

18. T. Stöcker et al., High-performance computing MRI simulations, Magn. Reson. Med. 64 (2010), 186-93.

19. G. Torheim et al., A simulator for teaching MR image contrast behavior magnetic resonance materials in physics, Biol. Med. MAGMA 2 (1994), 515-522.

20. J. E. Wilhjelm et al., An active learning approach to the physics of medical imaging, Med. Eng. Phys. 30 (2008), 607-614.

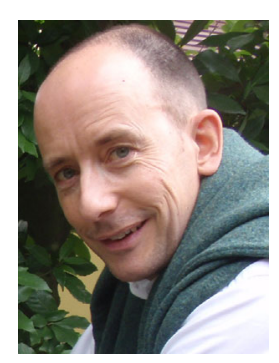

J. E. WiLhJELM received his MSc from the Technical University of Denmark (DTU) in 1986 and his $\mathrm{PhD}$ in Biomedical Engineering from the Biomedical Engineering Department at Worcester Polytechnic Institute, Massachusetts, USA in 1991. $\mathrm{He}$ is currently professor of Medical Imaging and Biomedical Engineering Education at Department of Electrical Engineering, DTU and has coauthored papers in both areas. In addition to teaching in medical imaging and biomedical instrumentation (the latter in collaboration with Engineering World Health), he is also director of the MSc in Biomedical Engineering, a joint educational program with the Faculty of Health and Medical Sciences, University of Copenhagen, Denmark.

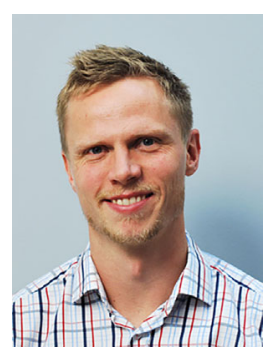

J. DuUn-Henriksen received the MS in Biomedical Engineering and Industrial $\mathrm{PhD}$ degrees from the Department of Electrical Engineering, Technical University of Denmark, in 2008 and 2013, respectively. His $\mathrm{PhD}$ was in collaboration with the company HypoSafe A/S now called UNEEG medical A/S and Copenhagen University Hospital. After he finished the $\mathrm{PhD}$, he continued in the company as a scientific researcher. In 2017, he won the People's Choice award at the annual Epilepsy Foundation Shark tank competition. $\mathrm{He}$ is now the Head of Epilepsy Research at UNEEG medical $\mathrm{A} / \mathrm{S}$ and has special responsibility within EEG data quality. In August 2018, he will start as a visiting researcher in the Richardson Lab at King's College London.

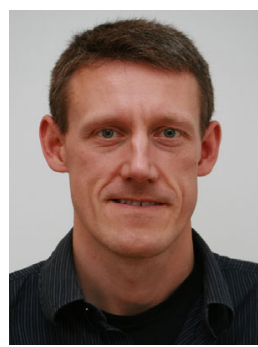

L. G. Hanson was educated in Physics, Math, and Computer Science at University of Copenhagen and received $\mathrm{PhD}$ in year 2000. He is associate professor at the Center for Magnetic Resonance (CMR) at the Technical University of Denmark (DTU), and senior researcher at the Danish Research Centre for Magnetic Resonance (DRCMR). His main research focus is on MRI acquisition technology. His interests include fast spectroscopic imaging, measurement of current-induced field changes in vivo for impedance tomography, and methods for handling system imperfections and patient motion. He has also published widely used educational software and texts focused on basic aspects of MRI.

How to cite this article: Wilhjelm JE, DuunHenriksen J, Hanson LG. A virtual scanner for teaching fundamental magnetic resonance in biomedical engineering. Comput Appl Eng Educ. 2018;1-13. https://doi.org/10.1002/cae.22028

\section{APPENDIX I}

\section{I.i Identical Introduction to Both Approaches (Compass} Magnetic Resonance)

The students are first given a rather short slide-based lecture introducing first a clinical example of MRI to trigger the interest. Nuclear magnetism for individual nuclei and 
ensembles are subsequently introduced by applying analogies to compass needles. Specifically, using a Java applet [9], it is shown and explained what happens to a compass when an external strong magnetic field is applied and when an orthogonal oscillating field is used for excitation. This is the basic magnetic resonance phenomenon illustrated using wellknown hardware.

\section{I.ii The Rest of the Classical Class}

After having demonstrated the continuous version, pulsed MR is introduced with interleaved excitation and sampling in presence of relaxation. Returning to slides, the difference between compass and nuclear dynamics is explained (vibration about vs. precession around the direction of the magnetic field). This difference is due to the atomic nuclei not only being magnetic but also having an associated angular momentum (they spin). This causes the oscillation frequency to be independent of amplitude, and hence points to the relevance of equation (1) that is also introduced. The students are required to read the course material [9] on these matters in advance. The slides are given to the students in advance, but with certain details missing (answers to questions posed during the lecture).

\section{I.iii Student Guide for Use With the MRI Virtual Scanner}

Instructions: The virtual scanner must be downloaded [4] and placed in a proper directory reachable by MATLAB. You will then need to type mri_demo at the MATLAB prompt (MATLAB version 2017 or later). You will then see a window as shown in Figure 1 (or an improved version). First take a look at the program and get acquainted with the user interface. Also, please read the text that appears when pressing Help and About buttons and also note the yellow tooltips that appear when moving the mouse cursor over a button. The questions below are intended to guide you to an understanding of the principles of Magnetic Resonance Imaging (MRI).

Questions to solve:

- Write down the name of the main components of an MR scanner as well as the responsibilities of each individual component.

- By operating the control panel of the scanner, reach a situation with the program, which creates a response from an object (leave gradients off for now).

- What is the difference in the received signal, when the type of object is changed?

- Try to identify how few components of the scanner that need to be active in order to show the principle of magnetic resonance (i.e., obtaining a response from the object in the scanner).

- Make a drawing of the signals that you see with correct horizontal axis.

- What does the signal represent?

- Write down parameters, equation(s) and their value for the above situation. Group the parameters logically (e.g., parameters related to the object, the MR scanner, etc.).

- What is needed to make the radio wave transmitter rotate the magnetization $90^{\circ}$ away from equilibrium, which gives maximum signal strength in the received signal?

- Try to make a diagram showing how the different parameters/components interact with the equation for the Larmor frequency and what determines the relaxation time.

- Try to obtain an image from a human. What happens to the image when you change the frequency of the transmitted pulse? And why?

Additional questions addressing slice selection can be posed if time allows, for example, regarding the relations between pulse duration/bandwidth, gradient strength, and slice thickness/position. 